\title{
Directed-Forgetting in Working Memory
}

\author{
Hannah Dames \& Klaus Oberauer \\ University of Zurich
}

\begin{abstract}
Author Note
This research was supported by funding from the Swiss Confederation as part of the Swiss Government Excellence Scholarships for Foreign Scholars and Artists to H. Dames, by the German Research Foundation, DFG [grant number RA1934/5-1] to M. Ragni, and by funding from the Swiss National Science Foundation (SNSF) to K. Oberauer [grant number 100014_179002]. We are grateful to Marie Jakob for her support when programming the experiments.

All experiments were preregistered prior to data collection. The data as well as materials and analysis scripts are publicly available on OSF (Dames \& Oberauer, 2021): https://osf.io/2vpfg/
\end{abstract}

\section{Correspondence:}

Hannah Dames

Department of Psychology

University of Zurich

Binzmühlestrasse 14/22

8050 Zürich, Switzerland

Email: hannah.dames@psychologie.uzh.ch

Note: This manuscript is a pre-print, and may not exactly replicate the final publication of this work. 


\begin{abstract}
How does the intent to remember or forget information affect working memory (WM)? To explore this question, in four experiments, we gauged the availability of the to-be-forgotten information directly. Participants remembered six words presented sequentially in separate frames. After each word offset, the frame turned either blue or orange, indicating a to-beremembered or to-be-forgotten word, respectively. In all experiments, consistently poor recognition performance for to-be-forgotten words and facilitation of to-be-remembered words demonstrated that intent has a strong impact on WM. These directed-forgetting effects are remarkably robust: They can be observed when testing the to-be-forgotten words up to four times (Experiment 1, $n=341$ ), for both item and binding memory (Experiment 3, $n=124$ ), and even when information has to be maintained in WM up to $5 \mathrm{~s}$ until the memory cue is presented (Experiment $2+4, n=302+321$ ). Our study establishes a new method to jointly study the effects of intent on WM content for both relevant and irrelevant information and provides evidence for directed-forgetting in WM. Our research suggests that a combination of two processes cause directed-forgetting in WM: One process reduces memory strength of earlier memory representations as a function of subsequently encoded events. Another process rapidly encodes or boosts memory strength only when the person intends to remember that information.
\end{abstract}

Keywords: directed-forgetting, working memory, removal 


\section{Introduction}

Forgetting has a bad reputation. It bothers us to accidentally forget a friend's birthday or a new PIN-code. Yet, forgetting is an important mechanism to deal with the vast amount of rapidly changing information we are confronted with. Working memory (WM) holds mental representations temporarily available for use in thought and action (Oberauer et al., 2018). Compared to long-term memory (LTM), WM has a limited capacity, so that WM content needs to be constantly updated to focus on current, relevant information. Therefore, there should be a mechanism that removes outdated information from WM (Lewis-Peacock et al., 2018).

Removing information from WM should have two consequences: freeing WM capacity for relevant information and impairing memory for the removed content. Evidence for removal from WM comes predominantly from studies demonstrating the first predicted effect. For instance, Oberauer (2018) found increased memory performance for still relevant memory items when, out of a set of words, some words were cued to be irrelevant (as compared to a baseline where all words were relevant). Evidence for the second prediction, showing that information removed from WM is difficult to retrieve, is rare (Muter, 1980; Williams et al., 2013).

By contrast, in the literature on directed-forgetting in episodic LTM, testing the to-beforgotten items by a surprise test is common, and forms the very basis for assessing the success of intentional forgetting (e.g., Bjork, 1970; Fawcett \& Taylor, 2008; Macleod, 1999; Popov et al., 2019). During a learning phase of such paradigms, either a list of items or each individual item is followed by a remember or forget instruction. A subsequent LTM test probes participants' memory for all items regardless of the memory instruction. Typically, LTM for to-beremembered items is higher than for to-be-forgotten ones (see Bjork et al., 1998). Researchers speculate that WM processes underly item-wise directed-forgetting in LTM (e.g., by stopping to 
process to-be-forgotten information in WM; Fawcett \& Taylor, 2012). If so, we should observe directed-forgetting not only in LTM but also in WM.

The first goal of our study was to develop and introduce a new task design, which combines the WM-removal-paradigm of Oberauer (2018) with a test of memory for removed WM contents, as it is usual in directed-forgetting studies of LTM. The new method was designed to jointly study the effects of removal on both relevant and irrelevant information in WM within one experiment. To investigate what happens with WM contents when cued to-be-forgotten, we tested their availability directly.

Our second goal was to test whether items are harder to remove from WM after they have been maintained for a longer time. This hypothesis was motivated by the assumption that representations in WM are initially fragile, before short-term consolidation establishes them more firmly (Ricker et al., 2018). The earlier a forget cue follows the to-be-forgotten item, the earlier short-term consolidation can be interrupted. This should make removing the item from WM easier.

Our third goal was to assess separately how directed-forgetting affects memory for the item itself, and memory for the item's relation to its context. According to the three-embeddedcomponents model of WM (Oberauer, 2009), the capacity-limited core component of WM maintains temporary item-context bindings, whereas item memory is maintained by activated LTM representations. Consequently, removal of contents from WM has been described as unbinding them from their contexts (Lewis-Peacock et al., 2018). Therefore, directed-forgetting in WM should reduce binding memory, but perhaps not item memory. Previous work gauged item memory for to-be-forgotten WM contents only indirectly through their tendency to interfere with retrieval of to-be-remembered contents. These studies have revealed that item memory long 
outlasts the time at which it became irrelevant (Monsell, 1978; Oberauer, 2001). Yet, one study reported reduced interference from to-be-forgotten items in an item-memory test (Festini \& Reuter-Lorenz, 2014). Here, we investigate the effect of directed-forgetting on item memory by measuring access to item information directly.

\section{The Present Experiments}

Participants remembered up to six words presented sequentially across a circular arrangement of frames. After the offset of each word, the frame turned either blue or orange, indicating a to-be-remembered or to-be-forgotten word, respectively. We varied the number of to-be-remembered-words (i.e., the set-size): There were two baseline conditions where all presented words were to-be-remembered, one with a large set-size (6 words) and one with a small set-size ( $3 \pm 1$ words). In the removal condition, three $( \pm 1)$ out of six words were cued tobe-forgotten, so that the number of remaining to-be-remembered words was equal to the small set-size baseline. To gauge memory for the to-be-forgotten words directly, on a small subset of trials we probed one of the to-be-forgotten words - on all other trials, only to-be-remembered frames were tested.

The removal hypothesis leads to two predictions: First, if to-be-forgotten information is removed from WM, it should be less accessible afterwards. Hence, performance in tests of to-beforgotten items should be lower than the to-be-remembered items of the same condition (directed-forgetting effects). Second, if information is removed from WM, it should not interfere with the still relevant words, resulting in facilitation effects for the to-be-remembered information. Therefore, performance for to-be-remembered words in the removal condition should be better than in the large set-size baseline. If to-be-forgotten items were completely 
removed from WM, performance of to-be-remembered words in the removal condition should be equal to the small set-size baseline.

We conducted four experiments that tested these two predictions within one experimental design. We consistently observed robust directed-forgetting effects for both item and binding memory, accompanied by facilitation effects for the still relevant information in WM. Delaying the forget cue for up to 5 seconds did not reduce directed-forgetting effects.

\section{Experiment 1}

The goal of Experiment 1 was to establish the feasibility of our new paradigm for jointly testing the two predictions from the removal hypothesis (facilitation and directed-forgetting effects). Gauging memory for to-be-forgotten words on a small subset of trials directly raised a methodological challenge as participants may start to ignore the forget cues (Williams \& Woodman, 2012). To assess how often we could test to-be-forgotten items without undermining the forget instruction, in Experiment 1, we varied the test frequency of to-be-forgotten words between participants (once, twice, or four times) and assessed the stability of directed-forgetting effects.

\section{Method}

Methods, hypotheses, and the data analysis procedures were preregistered and can be found online (https://osf.io/rqmw5, Dames \& Oberauer, 2021). There, we also report detailed information on the randomization procedure.

\section{Participants and Exclusion Criteria}

In total, 453 native English speakers completed the whole experiment via the web-based participation platform Prolific. After applying the following preregistered exclusion criteria, the final sample consisted of 341 participants $\left(M_{\mathrm{age}}=26.7, S D_{\mathrm{age}}=5.2\right.$, range $18-38$ years; $57.5 \%$ 
female, $42.5 \%$ male): We excluded participants with a mean error rate higher than $40 \%$ in the small set-size condition $(n=4)$, who reported having suspected that to-be-forgotten stimuli would be tested $(n=10)$, who stated that they strongly tried to remember the to-be-forgotten words $(n=5)$, or who had technical issues during completion $(n=3)$. Additionally, we excluded 90 participants that did not try to respond correctly on to-be-forgotten surprise recognition probes (see Experiment 2 for a solution to this problem).

We needed a relatively large sample because we were interested in recognition accuracy of to-be-forgotten items, which were rare in our design. The sample size was chosen based on prior experience with similar experiments (sample sizes of all subsequent experiments were based on the results of Experiment 1). As we used Bayesian analyses, we planned to increase the sample size if we found that some hypothesis tests were ambiguous (i.e., $1 / 3<\mathrm{BF}<3$; this criterion was preregistered). As all BFs for the directed-forgetting effects in WM (difference between the removal remember and the removal forget condition) were remarkably large in all experiments, we did not further increase our sample size.

All participants were naive to the purpose of the experiment and had normal or correctedto-normal vision. Participants signed an informed consent form prior to the study, and they were debriefed at the end. The study protocol is in line with the guidelines of the institutional ethics review board. Participants were randomly assigned to one of three between-subject groups (tobe-forgotten words were tested once: $n=124$, twice: $n=101$, four times: $n=116$ ).

\section{Material}

Frequent English nouns with a length of 4-5 letters served as stimuli. For every participant 396 words were randomly drawn from a pool of 600 words. The participants' task 
was to remember the words and recognize them in a recognition test using the left or right arrow key on their keyboard. The experiment was programmed using jsPsych (de Leeuw, 2015).

\section{Procedure}

Our new task design combined paradigms used to investigate item-method LTM directedforgetting (Bjork, 1970) and item-wise removal in WM (Oberauer, 2018). The trial structure is illustrated in Figure 1: Participants first saw six squared frames arranged on a circle $\left(60^{\circ}\right.$ apart from each other). The frames were presented in thin black outlines on a white background. After presentation of all six empty frames for $1 \mathrm{~s}$, six words were displayed one by one for $1 \mathrm{~s}$ each in a clockwise order across the circular arrangement of frames, starting on the top left at 10 o'clock. Immediately following each word offset, the frame of that word turned either blue or orange for $0.5 \mathrm{~s}$, indicating a to-be-remembered or to-be-forgotten word, respectively. Following the offset of each cue the frames remained empty for $0.5 \mathrm{~s}$ before the subsequent word was presented in the next frame. After all words have been displayed, and following a retention interval of $1 \mathrm{~s}$, a localrecognition probe was presented in one of the frames. Using left and right keypresses, participants indicated whether a presented word was the same as the one originally presented in that frame (match, 50\%) or not (mismatch, 50\%). A mismatch probe was always a word from another frame in the that trial. In this way, the local-recognition task tests memory for itemlocation bindings. We chose this test because WM capacity is primarily a limit on binding memory (Oberauer, 2019), and because removal has been described as un-binding item representations from their contexts (Lewis-Peacock et al., 2018). 


\section{Figure 1}

General Trial Structure

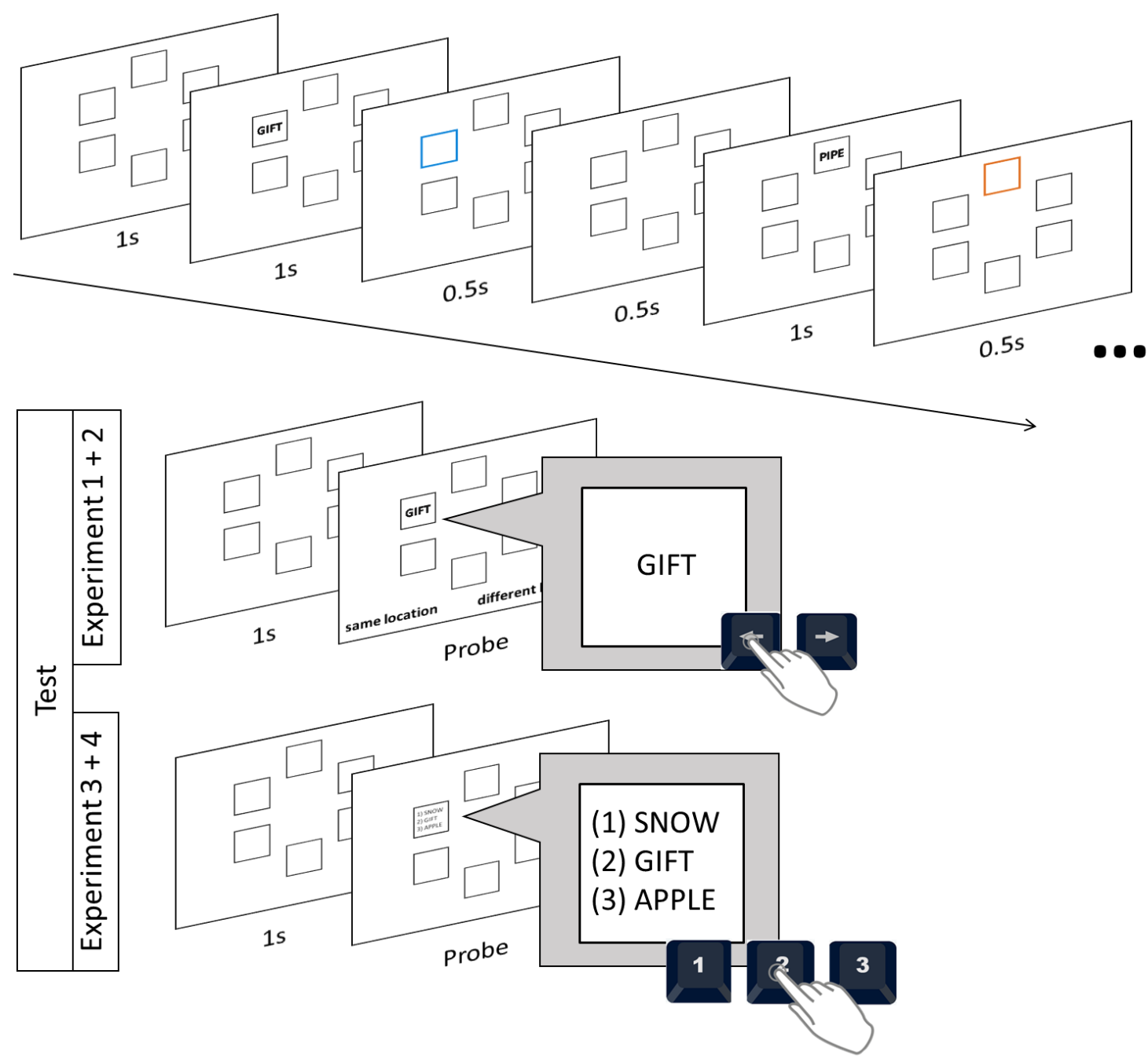

Set-size conditions. There were three set-size conditions. In the large set-size condition, participants were instructed to remember all six presented words. On those trials, every word was followed by a remember cue (i.e., a blue frame). In the small set-size condition, three randomly chosen words were replaced by "XXXX" (displayed in the same way as the words). "XXXX"stimuli were always followed by a forget cue (i.e., an orange frame). The other three words were followed by a remember cue. In the removal condition, three randomly chosen words were followed by a remember cue and the other three words were followed by a forget cue. There 
were 20 trials for each set-size condition, presented in random order (except for trials where a tobe-forgotten words was tested, see below).

For the large and the small set-size conditions, and the majority of trials of the removal condition the tested frame was randomly selected from one of the to-be-remembered words. A match probe was the word from that frame; a mismatch probe was a word from another to-beremembered frame in that trial. On very few trials of the removal condition, the local-recognition probe was presented in one of the to-be-forgotten frames to test participants' memory of one randomly selected to-be-forgotten word. How often a to-be-forgotten word was probed depended on the group participants were assigned to (see below). In these trials, a mismatch probe was a word from one of the other to-be-forgotten frames.

Test frequency of to-be-forgotten words. We varied the frequency with which the to-beforgotten words were tested (once, twice, or three times) between participants: $1 / 3$ of the participants received only one to-be-forgotten test in the very last trial $\left(\operatorname{trial}_{\mathrm{n}}=60\right)$ of the experiment. Whether the location of the recognition probe of this to-be-forgotten test matched the location of the original word position or not, was chosen at random with equal probability. Another third of the participants received a to-be-forgotten test twice within the experiment once after having completed $1 / 3$ of the experiment at trial ${ }_{n}=20( \pm 1$ trial; to reduce the chance that the trial with to-be-forgotten test can be predicted, these trial positions were shuffled by adding \pm 1 trial at random) and once after $2 / 3$ of the experiment at trial $\mathrm{n}_{\mathrm{n}}=40 \pm 1$. The remaining third of the participants received a to-be-forgotten test four times within the experiment - after having completed $1 / 5,2 / 5,3 / 5$, and $4 / 5$ of the experiment at trials trial $\mathrm{n}_{\mathrm{n}}=12,24,36$, and 48 (all \pm 1 trial). 
Instructions and Practice. The instructions stressed that participants should remember only the to-be-remembered words for the subsequent recognition test, and that they should try to forget the to-be-forgotten words. Participants were told that they should truly try to forget all words in an orange frame, as this would make it easier for them to remember the to-beremembered words. Following the instructions, participants had the chance to practice their task (two small set-size trials, two large set-size trials, and two removal trials with tests of a to-beremembered word, all presented in random order). In the practice block, participants received feedback ("correct"/ "incorrect") after each recognition decision. They received no feedback on their performance for the remainder of the experiment. After the practice block, participants had the option to reread the instructions and redo the practice block or proceed with the experiment. Next, the main experimental block followed.

Using post-experimental questions, we checked whether participants had any suspicion about the purpose of the experiment, to what extent they followed the task instructions (e.g., what they did when they saw an orange or blue frame), and whether they expected that the to-beforgotten words would be tested. Furthermore, participants indicated how often they noticed that to-be-forgotten words were probed and if so, how they responded on those occasions (i.e., if they tried to respond correctly on those trials or not). For instance, because the test of to-be-forgotten words came as a surprise, some participants reported that they always indicated that the to-beforgotten probes were a mismatch regardless of whether they could actually remember the to-beforgotten words or not. By excluding these participants, we tried to minimize the risk of overestimating directed-forgetting effects. 


\section{Data Analysis}

We analyzed the data of all our experiments with Bayesian mixed models (for an overview of mixed models see Baayen et al., 2008; Judd et al., 2012). We analyzed the correctness of responses using Bayesian generalized linear mixed models (BGLMMs) assuming a Bernoulli data distribution predicted by a linear model through a logistic link function. RTs were log-transformed and analyzed using Bayesian linear mixed models (BLMMs) assuming a Gaussian data distribution. The $\mathrm{B}(\mathrm{G}) \mathrm{LMMs}$ were estimated using the $R$ package brms (Bürkner, 2018). All our models included random intercepts for participants. For the regression coefficients of the accuracy analyses, we used moderately informative Cauchy priors with a scale of 0.353 and a mean of 0 (recently proposed default priors for logistic models; Oberauer, 2019). For random effects, we used non-negative weakly informative priors (half student t-prior with three degrees of freedom and a scaling parameter of 2.5). For the RT analyses, the brms default settings for priors were used. All categorical predictors were coded as sum-to-zero contrasts and continuous predictors were mean-centered. In all models, to control for effects of learning and/or fatigue throughout the experiment, we included the continuous predictor trial (trial number of a given trial) in all our models.

We estimated the posteriors by sampling parameter values using the No-U-Turn Sampler (NUTS, an extension of the Hamilton Monte Carlo sampling method) as implemented in Stan (Carpenter et al., 2017). We sampled through four independent Markov chains with 50,000 iterations each (2,000 warm up each). To investigate convergence, we inspected Rhat values (ratio of between-chain variance to within-chain variance). Rhat values were $\leq 1.01$ for all parameters in every model. Additionally, we inspected the chains for convergence visually. 
We computed Bayes Factors (BFs, calculated through the bridge sampler Gronau et al., 2018) to estimate the strength of evidence for the null and the alternative hypothesis. To this end, we fitted two competing, nested models for each hypothesis: One including the effect/factor of interest and one that did not. We then compared the two models to calculate the evidence for/against the null/alternative hypothesis. Generally, a BF larger than 3 can be considered as substantial evidence for one hypothesis over the other. For instance, a $\mathrm{BF}_{10}$ of 3 would indicate that the data are 3 times more likely under the alternative hypothesis than under the null hypothesis.

To test whether the likelihood to correctly identify a recognition probe as a match/mismatch for tests of to-be-forgotten words was close to chance, we compared two “intercept-only"-BGLMMs on the correctness of participants' responses on these trials. One model included a free parameter for the intercept. For the other model the intercept parameter was fixed at chance-level performance. We then tested whether the model including the intercept was superior to the model where the intercept was fixed.

\section{Results}

Mean RTs and accuracy rates as a function of condition and frequency of to-be-forgotten tests are displayed in Figure 2A. We evaluated differences between specific conditions (large and small set-size baseline, and tests of to-be-remembered and to-be-forgotten words from the removal condition) on participants' recognition accuracy and RTs. At the same time, we investigated how these effects changed depending on the relative frequency of preceding tests of to-be-forgotten words (i.e., the ratio of preceding to-be-forgotten test trials to preceding trials). 


\section{Figure 2}

Mean accuracy rates and reaction times (RTS) across set-sizes and conditions of $(A)$ Experiment 1 and (B) Experiment 2
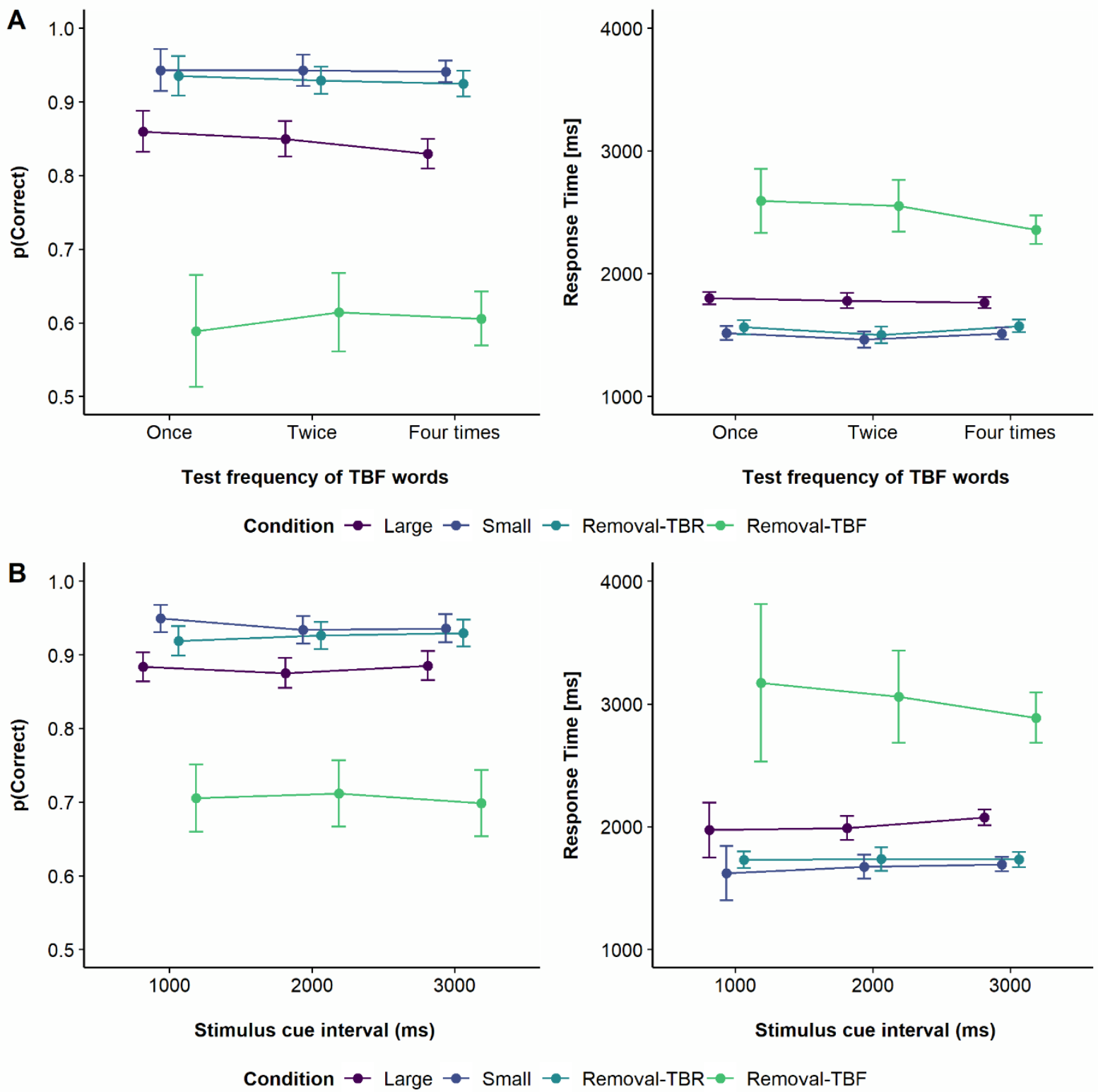

Note. Error bars represent the within subject $95 \%$ confidence intervals. $\mathrm{TBR}=$ to-be-remembered, $\mathrm{TBF}=$ to-be-forgotten 


\section{The Availability of To-Be-Forgotten Items}

One of our main interests lies in the availability of to-be-forgotten items at test. To estimate directed-forgetting in WM, we compared performance of to-be-forgotten and to-beremembered trials for the removal condition. As predicted, the likelihood to correctly identify a recognition probe as a match/mismatch was substantially lower for to-be-forgotten than for tobe-remembered words in the removal condition $\left(\mathrm{BF}_{10}=1.3 \times 10^{117}\right.$; evidence in favor of a model including the main effect of memory cue compared to a model excluding the effect). Furthermore, in the removal condition, RTs were larger for tests of to-be-forgotten than to-beremembered words $\left(\mathrm{BF}_{10}=7.8 \times 10^{200}\right)$. Hence, participants showed substantial directedforgetting effects in recognition performance and RTs. Still, the recognition performance of tobe-forgotten words was above chance level $\left(50 \%\right.$; $\left.\mathrm{BF}_{10}=851435\right)$.

\section{Effects of Facilitation}

For the analysis of facilitation effects for to-be-remembered WM content, we only analyzed trials in which to-be-remembered items were tested, and that were not preceded by any to-be-forgotten tests. As predicted, the probability for a correct response for to-be-remembered items was higher in the removal condition than in the large set-size baseline $\left(\mathrm{BF}_{10}=1.2 \times 10^{26}\right)$. RTs for to-be-remembered words were also faster in the removal condition than in the large setsize baseline $\left(\mathrm{BF}_{10}=2.2 \times 10^{69}\right)$. The evidence concerning an accuracy difference between the removal condition and the small set-size baseline was ambiguous $\left(\mathrm{BF}_{10}=1.0\right)$. When including all to-be-remembered trials (regardless of the frequency of the preceding tests of to-be-forgotten words) in the analyses, we found evidence for a difference between the removal condition and the small set-size baseline $\left(\mathrm{BF}_{01}=5.8\right)$. In addition, $\mathrm{RT}$ s for to-be-remembered words were larger in the removal condition than in the small set-size baseline $\left(\mathrm{BF}_{10}=100.5\right)$. As can be seen 
from Figure 2A, the performance differences between to-be-remembered words from the removal condition and the small set-size baseline condition were relatively small compared to the strong facilitation effects when comparing performance to the large set-size baseline condition.

\section{The Influence of How Often To-Be-Forgotten Words are Tested}

In general, we did not find any indication that the relative frequency of preceding tests of to-be-forgotten words (relative to all preceding trials) influenced the reported results (see Figure 2A). All BFs testing the interaction between condition and test frequency of to-beforgotten words yielded evidence against such an interaction or were ambiguous (large set-size vs. removal remember: $\mathrm{BF}_{01}=3.1$; small set-size vs. removal remember: $\mathrm{BF}_{01}=1.8$; removal remember vs. forget: $\mathrm{BF}_{01}=1.5$ ). The same was the case for RTs (large set-size vs. removal remember: $\mathrm{BF}_{01}=8.8$; small set-size vs removal remember: $\mathrm{BF}_{01}=1.1$; removal remember vs . forget: $\left.\mathrm{BF}_{01}=1.7\right)$.

To conclusively test whether we could measure directed-forgetting in WM even when testing the to-be-forgotten items up to four times, we compared the removal remember condition to the removal forget condition in the participant group that was tested four times. As expected, also for this group of participants, our model comparison yielded substantial evidence for a main effect of condition and hence, for directed-forgetting in $\mathrm{WM}\left(\mathrm{BF}_{10}=1.3 \times 10^{55}\right)$.

To summarize, in Experiment 1, very low recognition performance for to-be-forgotten words showed that to-be-forgotten items were no longer available in WM-even when the to-beforgotten words were tested four times. Consequently, recognition performance for to-beremembered words was higher than for to-be-forgotten words resulting in a substantial directedforgetting effect in WM. Furthermore, across all test frequencies, participants performed better in 
the removal remember condition than in the large set-size baseline, thus demonstrating a facilitation effect for to-be-remembered items.

\section{Experiment 2}

The aim of Experiment 2 was to replicate the findings of Experiment 1 while testing the boundary conditions of directed-forgetting in WM as measured by the new method. We analyzed whether holding an item in WM longer made it harder to subsequently remove it, as has been observed for directed-forgetting in LTM (Hourihan \& Taylor, 2006). Their experiments showed that instructed forgetting was less effective when the forget cue appeared at a longer delay after the to-be-forgotten item. Here we ask whether that is true also for directed-forgetting in WM. For this, we varied the time between the offset of each word and the onset of the memory cue (stimulus-cue-interval, SCI: 1s, 2s, or 3s) within participants.

\section{Method}

Methods, hypotheses, and the data analysis procedures were preregistered and can be found online (https://osf.io/59c3z, Dames \& Oberauer, 2021).

\section{Participants}

The same preregistered inclusion and exclusion criteria as in Experiment 1 were applied and we only recruited participants that had not participated in the previous experiment. From the initial sample $(N=353), 51$ participants had to be excluded, resulting in a final sample size of $n=302$ participants $\left(M_{\text {age }}=27.2, S D=4.7\right.$, range: $18-35$ years; $54.6 \%$ female, $44.4 \%$ male, $1 \%$ diverse). Probably due to our improved procedure (see below), only 18 participants had to be excluded because they did not know how to respond correctly on trials testing to-be-forgotten items. 


\section{Material and Procedure}

Stimuli and procedure were identical to Experiment 1 with the following exceptions:

First, memory of to-be-forgotten words was tested three times for all participants (on trials 16, 36 , and 56; \pm 1 trial; one match and two mismatch trials or two match and one mismatch trial). Second, following each word offset, all frames remained empty for either $1 \mathrm{~s}, 2 \mathrm{~s}$, or $3 \mathrm{~s}$ depending on the current SCI condition. Only after the SCI, the frame turned either blue or orange for indicating a to-be-rememberd or to-be-forgotten word, respectively. For each participant, there were 18 trials for each of the two baseline conditions, 18 trials of the removal condition with a test of the to-be-remembered item, and three with a test of the to-be-forgotten trial. Within each of these four conditions, an equal number was randomly assigned to each SCI level. All trials were presented in a random order.

Third, we fine-tuned our method to rule out two alternative explanations for our previous results. In Experiment 1, the test of to-be-forgotten words came as a surprise. Consequently, we had to exclude a large number of participants $(n=90)$ who reported that they were unsure how to respond correctly on those trials. Although we excluded those participants from our analyses in Experiment 1, possibly, due to the surprise test nature of the to-be-forgotten words, directedforgetting effects were generally overestimated. To rule out this possibility, at the beginning of Experiment 2 (and all subsequent experiments), we informed participants that on very few occasions their memory for to-be-forgotten words would be tested. Participants were instructed to do their best to remember the to-be-forgotten words. They were told that they should nevertheless truly try to forget all words in an orange frame, as this would make it easier for them to remember the to-be-remembered words, and therefore put them - on average - at an 
advantage. Participants were additionally instructed not to use any external help (e.g., use pen and paper).

In Experiment 1, the number of to-be-forgotten items was always the same for the removal condition. At the end of each trial, participants may have been able to predict the subsequent memory cue(s) prior to item presentation, potentially resulting in stronger directedforgetting effects, because later to-be-forgotten words may not have been encoded into WM at all. Therefore, in Experiment 2 (and all subsequent experiments), we varied the frequency of tobe-remembered words in the removal condition and the small set-size baseline to be either two, three, or four. Hence, in the two baselines, participants remembered either all six (large set-size) or only three \pm 1 (small set-size) words. In the removal condition, three $( \pm 1)$ out of six words were cued to-be-forgotten. Thereby, we ensured that participants could not predict the nature of the upcoming memory cues.

\section{Statistical Analyses}

The statistical analysis procedures were identical to Experiment 1 except the following: In a first step, we investigated the influence of the SCI on each set-size contrast. In addition, to test the availability of to-be-forgotten information directly, for removal forget trials only, we compared one model including the factor SCI against an intercept-only model. Because we found no indication that SCI influenced performance in any of the models, we analyzed the availability of to-be-forgotten items as well as effects of facilitations including all trials regardless of the SCI (same procedure as in Experiment 1). For reasons of readability, we start reporting the results with this latter analyses. We had preregistered the plan to add the number of to-be-remembered items in the small set-size baseline and the removal condition as a covariate in some models. As 
we found substantial directed-forgetting effects regardless of this predictor, we did not further consider it in any of the subsequent analyses.

\section{Results}

Mean RTs and accuracy rates can be taken from Figure 2B.

\section{The Availability of To-Be-Forgotten Items}

Replicating the results of Experiment 1, the likelihood to correctly identify a recognition probe as a match/mismatch was substantially lower in the removal forget condition than in the removal remember condition $\left(\mathrm{BF}_{10}=1.1 \times 10^{38}\right)$. Furthermore, $\mathrm{RTs}$ were larger in the removal forget condition than in the removal remember condition $\left(\mathrm{BF}_{10}=1.4 \times 10^{87}\right)$. Recognition performance of to-be-forgotten words was again above chance level $\left(50 \% ; \mathrm{BF}_{10}=4.0 \times 10^{29}\right)$.

\section{Effects of Facilitation}

In Experiment 1, we found no indication that the relative frequency of previous to-beforgotten tests affected the facilitation of to-be-remembered words. Hence, in the subsequent analyses, we included all trials regardless of whether they were preceded by any test for to-beforgotten words or not. The probability for a correct response was again substantially higher in the removal remember condition than in the large set-size baseline $\left(\mathrm{BF}_{10}=2.3 \times 10^{12}\right)$. Likewise, RTs were faster in the removal remember condition than in the large set-size baseline $\left(\mathrm{BF}_{10}=1.1 \times 10^{92)}\right.$. This time, recognition performance in removal remember trials was poorer than in the small set-size baseline $\left(\mathrm{BF}_{10}=4.2\right)$ and also RTs were larger in the removal remember condition than in the small set-size baseline $\left(\mathrm{BF}_{10}=50460.4\right)$. Like in Experiment 1 , those differences were rather small (see Figure 2B). 


\section{Testing the Influence of the Stimulus Cue Interval}

As illustrated in Figure 2B, we did not find any indication that the SCI influenced the results. All BFs testing the interaction between set-size and SCI yielded evidence against such an interaction (large set-size vs. removal remember: $\mathrm{BF}_{01}=7.2$; removal remember vs. forget: $\mathrm{BF}_{01}=3.1$ ); one was ambiguous (small set-size vs. removal remember: $\mathrm{BF}_{01}=1.5$ ). The same was the case for RTs (large set-size vs. removal remember: $\mathrm{BF}_{01}=29.0$; small set-size vs removal remember: $\mathrm{BF}_{01}=3.0$; removal remember vs. forget: $\mathrm{BF}_{01}=22.0$ ).

In summary, we replicated the directed-forgetting and facilitation effects in WM across all SCI levels.

\section{Experiment 3}

Experiments 1 and 2, we established a direct way to assess the availability of to-beforgotten information in WM, demonstrating robust directed-forgetting of memory for bindings between items and their location. Experiment 3 tested whether directed-forgetting affects not only memory for item-context bindings but also item memory itself. For this, we expanded the local-probe recognition test used in our previous experiments using a three-alternative forced choice (3AFC) test.

\section{Method}

Methods, hypotheses, and the data analysis procedures were preregistered and can be found online (https://osf.io/spu29, Dames \& Oberauer, 2021).

\section{Participants}

Inclusion and exclusion criteria were the same as before. From the initial sample $(N=150), 26$ participants were excluded resulting in a final sample size of $n=124\left(M_{\text {age }}=25.9\right.$, $S D=4.6$, range: $18-35$ years; $57.3 \%$ female, $41.1 \%$ male, $1.6 \%$ ). 


\section{Material and Procedure}

In Experiment 3, we expanded the local-probe recognition test used in our previous experiments using a three-alternative forced choice (3AFC) test (Figure 1). The same English nouns as in Experiment 1 and 2 served as stimuli. This time, however, for every participant 462 words were randomly drawn from a pool of 600 words.

The trial structure was similar to Experiment 2, but the SCI was always 0s. Instead of the single local recognition probe, a $3 \mathrm{AFC}$ test was used. In one randomly selected to-beremembered frame, three test words were presented: (1) one word matched the previously presented word in that frame ("old-match"), (2) one word belonged to another to-be-remembered frame in that trial ("old-mismatch"), (3) and one word was new. Using the keys [1],[2], and [3] participants' task was to select the word that was the same as the one originally presented in the frame ("old-match"). The order of these three words was random on each trial. On the three removal forget trials, the $3 \mathrm{AFC}$ test was presented in one of the to-be-forgotten frames (with the old-mismatch word being also a to-be-forgotten word).

\section{Results}

Participants' likelihood to select an old over a new word [p(old)] served as a measure for item memory. Binding memory was measured by participants' likelihood to select the match word, given they selected an old word [p(match|old)]. If participants had item memory for the tobe-forgotten words, participants should be able to correctly identify the two to-be-forgotten words as "old" words and hence, rarely select the "new" words at test. Yet, if removal weakened item-context memory, they should struggle to correctly discriminate between the old match and mismatch word. 
Mean accuracy rates can be taken from Figure 3A. The statistical analysis procedures were the same as in Experiment 2 but were conducted for p(old) and p(match|old) separately. For the 3AFC, we did not analyze RTs.

\section{Figure 3}

$P($ old $)$ and $p$ (match $\mid$ old $)$ Across Set-Sizes and Conditions of (A) Experiment 3 and (B)

\section{Experiment 4}
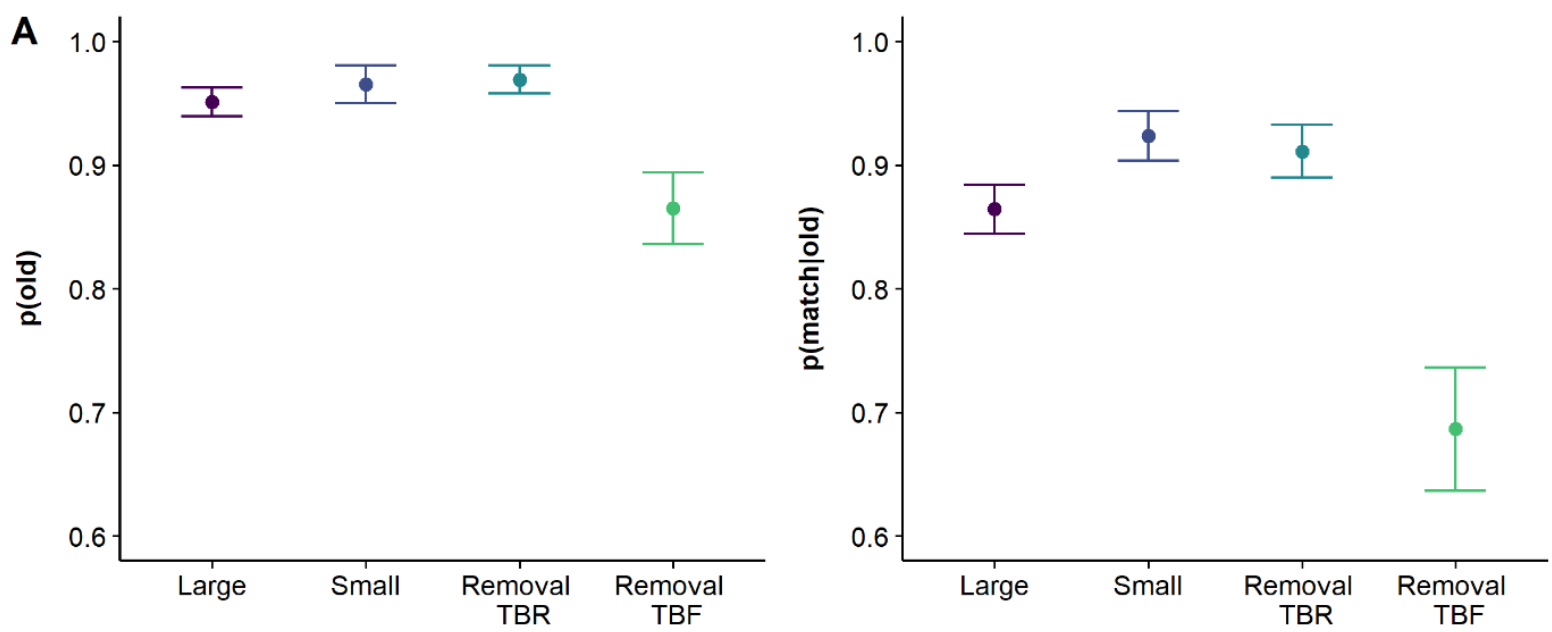

Condition

Condition

Condition $\rightarrow$ Large $\rightarrow$ Small $\rightarrow$ Removal-TBR $\rightarrow-$ Removal-TBF
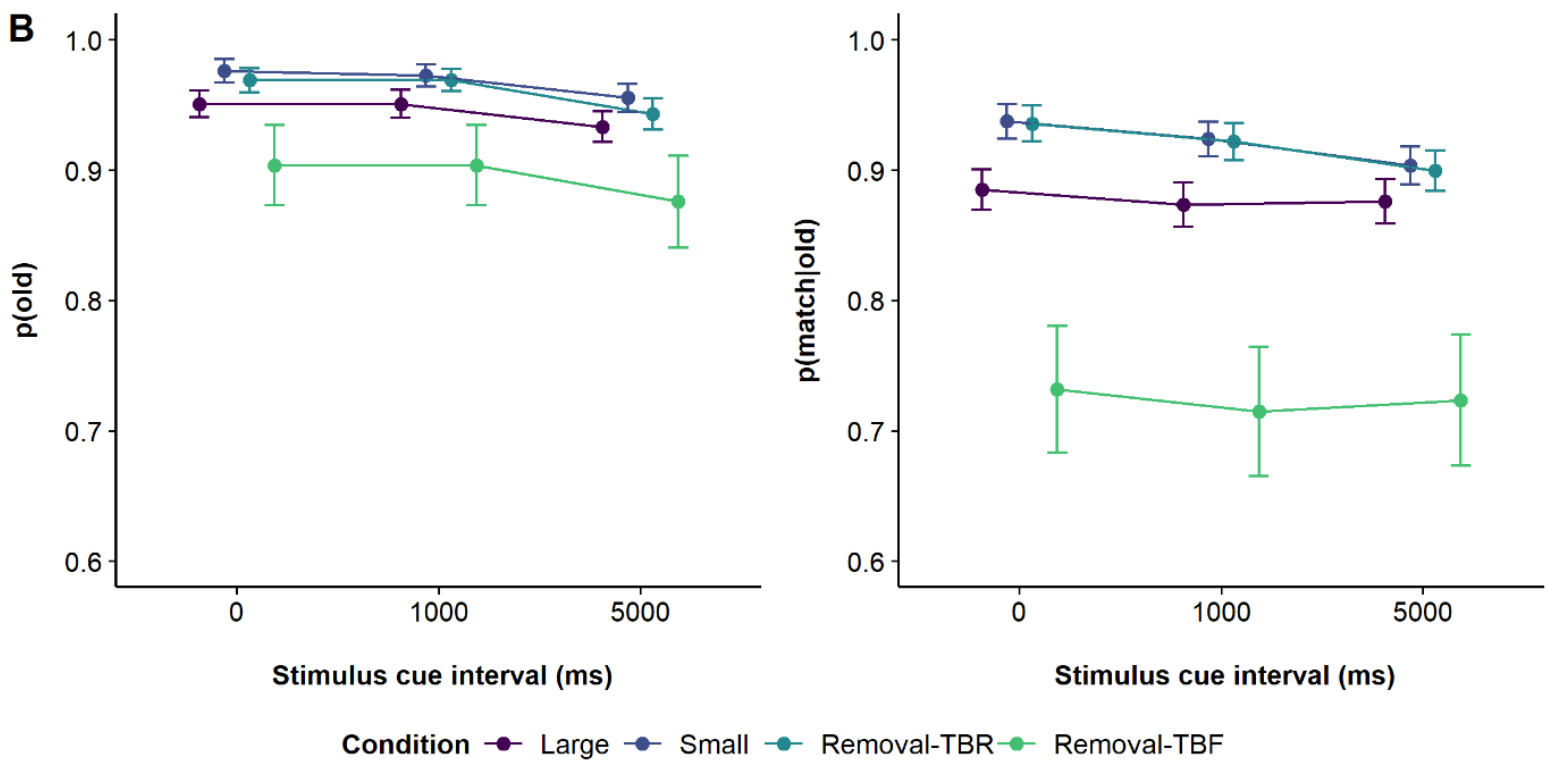

Note. Error bars represent the within subject 95\% confidence intervals. TBR $=$ to-be-remembered, $\mathrm{TBF}=$ to-be-forgotten 


\section{The Availability of To-Be-Forgotten Items}

$\mathrm{P}(\mathrm{old})$ in the removal condition was substantially lower for to-be-forgotten than for to-beremembered words $\left(\mathrm{BF}_{10}=6.2 \times 10^{6}\right)$ indicating a directed-forgetting effect for item-memory. Furthermore, p(old) for to-be-forgotten words was above chance level (66.67\%;

$\left.\mathrm{BF}_{10}=1.3 \times 10^{15}\right)$. We again observed a directed-forgetting effect in binding memory: $\mathrm{p}($ match|old) was substantially lower for to-be-forgotten than for to-be-remembered words $\left(\mathrm{BF}_{10}=5.5 \times 10^{29}\right)$. Furthermore, $\mathrm{p}($ match|old $)$ for to-be-forgotten words was above chance level $\left(50 \% ; \mathrm{BF}_{10}=1166108.4\right)$

\section{Effects of Facilitation}

Both item memory $\left(\mathrm{BF}_{10}=11.3\right)$, as measured by $\mathrm{p}(\mathrm{old})$, as well as binding memory $\left(\mathrm{BF}_{10}=167789\right)$, as measured by $\mathrm{p}($ match|old $)$, were higher in the removal remember condition than in the large set-size baseline. $\mathrm{P}($ old $)$ did not differ between the removal remember condition and the small set-size baseline $\left(\mathrm{BF}_{01}=4.7\right)$. For $\mathrm{p}($ match $\mid \mathrm{old})$, evidence concerning a difference between the removal condition and the small set-size condition was ambiguous $\left(\mathrm{BF}_{01}=2.7\right)$.

To summarize, the results of Experiment 3 demonstrate directed-forgetting not only for binding but also for item memory. In addition, we observed the typical facilitations effects for tobe-remembered WM content for both item and binding memory.

\section{Experiment 4}

In Experiment 3, we found evidence for directed-forgetting in WM not only for binding but also for item memory. In Experiment 4, we tested again whether delaying the forget cue led to a more robust representation in WM that is harder to remove. To make sure that we did not 
miss a subtle effect of cue delay in Experiment 2, here we varied the SCI over a broader range, from 0 to $5 \mathrm{~s}$.

\section{Method}

Methods, hypotheses, and the data analysis procedures were preregistered and can be found online (https://osf.io/hkc4u, Dames \& Oberauer, 2021).

\section{Participants}

The same inclusion and exclusion criteria as in Experiment 3 were applied. From the initial sample $(N=398), 77$ participants were excluded, resulting in a final sample size of $n=321\left(M_{\text {age }}=26.7, S D=5.2\right.$, range: $18-35$ years; $57 \%$ female, $41.4 \%$ male, $1.6 \%$ diverse $)$.

\section{Material and Procedure}

Stimuli and procedure were identical to Experiment 3 with the only difference that following each word offset, all frames remained empty for either $0 \mathrm{~s}, 1 \mathrm{~s}$, or $5 \mathrm{~s}$ depending on the current SCI condition (varied within-subjects as in Experiment 2)

\section{Results}

Mean accuracy rates can be taken from Figure 3B.

\section{The Availability of To-Be-Forgotten Items}

Replicating the results of Experiment 3, both item memory $\left(\mathrm{BF}_{10}=2.1 \times 10^{10}\right)$, as measured by $\mathrm{p}(\mathrm{old})$, as well as binding memory $\left(\mathrm{BF}_{10}=3.5 \times 10^{45}\right)$, as measured by $\mathrm{p}$ (match|old), were substantially lower for to-be-forgotten than for to-be-remembered words in the removal condition. Furthermore, both $\mathrm{p}($ old $)$ for to-be-forgotten words $\left(\mathrm{BF}_{10}=5.9 \times 10^{52}\right)$ as well as $\mathrm{p}\left(\right.$ match|old) for to-be-forgotten words $\left(\mathrm{BF}_{10}=8.4 \times 10^{27}\right)$ were above chance level. 


\section{Effects of Facilitation}

Item memory ( $\left.\mathrm{p}(\mathrm{old}), \mathrm{BF}_{10}=195.6\right)$, as well as binding memory ( $\mathrm{p}$ (match $\mid$ old $)$,

$\mathrm{BF}_{10}=1.4 \times 10^{12}$ ) for to-be-remembered words were higher in the removal condition than in the large set-size. Evidence for a difference in p(old) between the removal condition and the small set-size baseline was ambiguous $\left(\mathrm{BF}_{10}=1.6\right) . \mathrm{P}($ match|old $)$ was higher in the small set-size baseline than in the removal condition $\left(\mathrm{BF}_{10}=9.4\right)$. Again, this difference was relatively small (see Figure 3B).

\section{The Influence of the Stimulus Cue Interval}

For all of the above reported comparisons, we investigated how the effects between setsizes changed depending on the SCI interval (0s, 1s, or 5s). Different from Experiment 2, we ran separate comparisons between the SCI levels of 0s vs. 1s and 1s vs. 5s. Thereby we could pinpoint the impact of the SCI on the effectiveness of removal for very short (0s vs. 1s) and fairly long (1s. vs. 5s) durations. Hence, we tested the interaction between condition and SCI levels for each of these two contrasts separately. As illustrated in Figure 3B, we did not find any indication that the SCI influenced the strength of the condition effects. All BFs testing the interaction between condition and SCI contrasts yielded evidence against such an interaction or were ambiguous (see Table 1). 


\section{Table 1}

Bayes Factors Against the Interaction between SCI and Condition in Experiment 4

\begin{tabular}{|c|c|c|c|c|}
\hline \multirow{2}{*}{$\begin{array}{l}\text { Interaction between } \\
\text { SCI and effect of: }\end{array}$} & \multicolumn{2}{|c|}{$\mathrm{p}(\mathrm{old})$} & \multicolumn{2}{|c|}{$\mathrm{p}($ match $\mid$ old $)$} \\
\hline & Os vs. 1s & $1 \mathrm{~s}$ vs. $5 \mathrm{~s}$ & Os vs. 1s & 1s vs. $5 \mathrm{~s}$ \\
\hline $\begin{array}{l}\text { large set-size vs. } \\
\text { removal remember }\end{array}$ & $\mathrm{BF}_{01}=4.0$ & $\mathrm{BF}_{01}=3.2$ & $\mathrm{BF}_{01}=9.7$ & $\mathrm{BF}_{01}=0.7$ \\
\hline $\begin{array}{l}\text { small set-size vs. } \\
\text { removal remember }\end{array}$ & $\mathrm{BF}_{01}=3.3$ & $\mathrm{BF}_{01}=6.1$ & $\mathrm{BF}_{01}=8.8$ & $\mathrm{BF}_{01}=8.0$ \\
\hline $\begin{array}{l}\text { removal remember vs. } \\
\text { removal forget }\end{array}$ & $\mathrm{BF}_{01}=3.2$ & $\mathrm{BF}_{01}=4.9$ & $\mathrm{BF}_{01}=3.6$ & $\mathrm{BF}_{01}=4.4$ \\
\hline removal forget & $\mathrm{BF}_{01}=3.8$ & $\mathrm{BF}_{01}=2.3$ & $\mathrm{BF}_{01}=4.6$ & $\mathrm{BF}_{01}=4.9$ \\
\hline
\end{tabular}

Note. $\mathrm{SCI}=$ stimulus cue interval.

To summarize, we observed directed-forgetting and facilitation effects for both binding and item memory in WM across all SCI levels.

\section{Effects of Directed-Forgetting over Serial Position}

Taken together, Experiments 1-4 show robust directed-forgetting effects in WM. Yet, in all experiments, recognition performance of to-be-forgotten words was above chance level. This finding raises the question which to-be-forgotten words were still in $\mathrm{WM}$ at the time of test. We have reason to assume that particularly later-presented to-be-forgotten words may still be in WM (potential processes causing this pattern of results are discussed in the subsequent section): In a previous study using an item-wise LTM directed-forgetting procedure, Lee (2013) found that participants recalled later-presented to-be-forgotten words better than those presented earlier in a study list. Although Lee (2013) argued that these words were presumably still held in short-term memory at test, this assumption has not been directly tested. A recent study by Oberauer and Greve (in press) supports the notion that directed-forgetting effects in WM may differ as a function of serial position: Whereas memory for to-be-remembered lists showed the usual 
primacy and recency effects, recall of to-be-forgotten lists showed predominantly a recency effect.

In the following, we investigated how recognition performance changed as a function of serial position in Experiments 1-4. Figure 4 illustrates the resulting serial position curves. In all experiments, binding memory for to-be-remembered words in the large set-size was better for both early and later-presented words of a list (as evident in the typical bowed shape exhibiting both primacy and recency). In contrast, binding memory for to-be-forgotten words was particularly weak for early presented words (except for Experiment 1; but see Experiment 2 on issues that were resolved in our subsequent experiments). Furthermore, the serial position curves show a strong recency effect for binding memory of to-be-forgotten words. That is, starting from the middle part of the list, binding memory for to-be-forgotten words gradually increased over serial position.

In sum, in line with Lee (2013) and Oberauer \& Greve (in press), the serial position curves reveal no primacy but a strong recency effect for to-be-forgotten words. The enhanced memory for later-presented to-be-forgotten words suggests that these words were still held in WM. 


\section{Figure 4}

Serial Position Curved from (A, left) Experiment 1, (A, right) Experiment 2, (B) Experiment 3, and $(C)$ Experiment 4
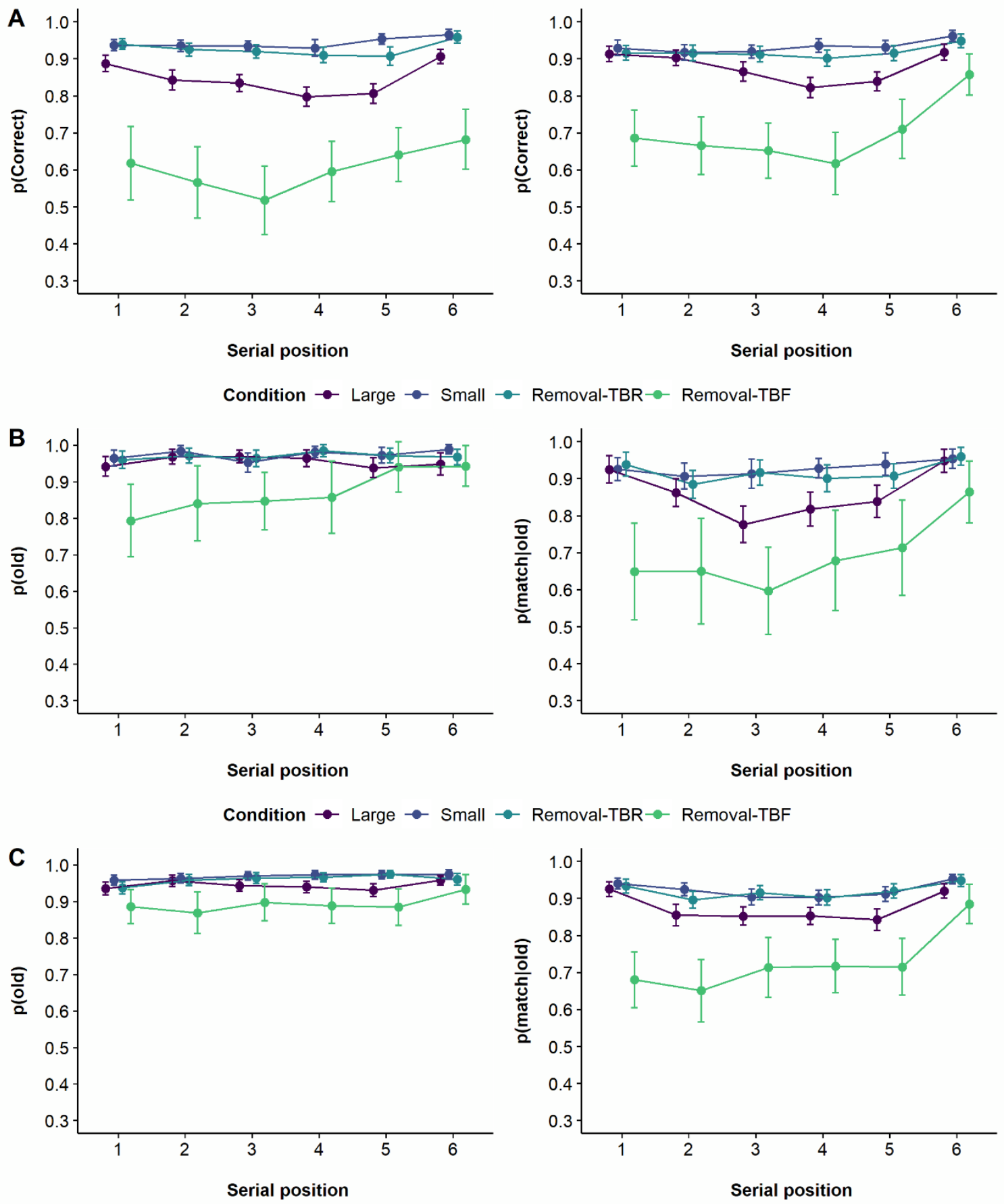

Condition $\rightarrow$ Large $\rightarrow$ Small $\rightarrow$ Removal-TBR $\rightarrow-$ Removal-TBF

Note. Error bars represent the within subject $95 \%$ confidence intervals. $\mathrm{TBR}=$ to-be-remembered, $\mathrm{TBF}=$ to-be-forgotten 


\section{Discussion}

The present study introduces a new method to jointly study the effects of directedforgetting on memory for both relevant and irrelevant information in WM. Across a series of four experiments, instructing participants to forget some words in WM resulted in very low item and binding memory for these words, accompanied by facilitation effects for the remaining to-beremembered WM content. These results demonstrate unambiguously that the to-be-forgotten words were no longer available in WM, and provide direct evidence for removal as a process for controlling the contents of WM (Ecker et al., 2014; Lewis-Peacock et al., 2018). They also converge with work revealing reduced interference of to-be-forgotten information in WM (Festini \& Reuter-Lorenz, 2013, 2014). By demonstrating both indicators of removal within one task, we confirm that directed-forgetting in WM goes hand in hand with facilitating memory for the remaining WM content.

We show that directed-forgetting weakened not only memory for item-context bindings but also memory for the items themselves. If WM is limited for the maintenance of temporary bindings but not item memory (Oberauer, 2019), then the intention to remember or forget affects not only the strength of bindings in WM but also that of item memory in episodic LTM. Against this assumption, Oberauer and Greve (in press) recently argued that because LTM has unlimited capacity, it records information regardless of intention. Our results challenge one of the two assumptions, suggesting that LTM is sensitive to intention or that WM contributes to measures of item memory.

\section{Which Processes Cause the Directed-Forgetting Effects in WM?}

We consider seven processes underlying directed-forgetting in WM (see Figure 5). They can be subdivided into explanations that involve the downregulation or loss of the to-be- 
forgotten information (e.g., Anderson \& Hanslmayr, 2014; Fawcett \& Taylor, 2008), and those that involve the upregulation or strengthening of the to-be-remembered information (e.g., MacLeod, 1975).

\section{Figure 5}

Given our Results: Suggested Processes Causing Directed-Forgetting in Working Memory.
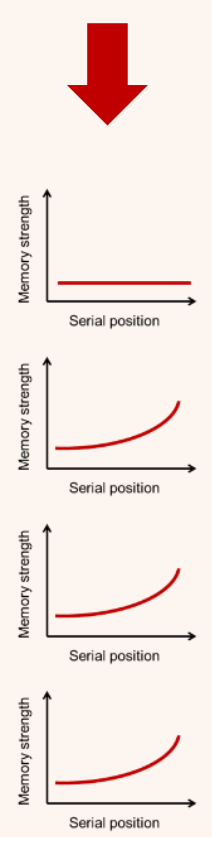

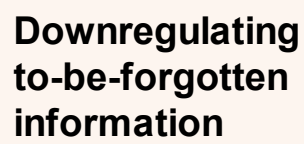

information

Selective removal

Decay

Automatic updating

Context-drift
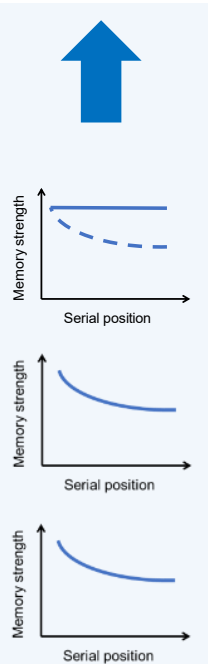

Attentional boost

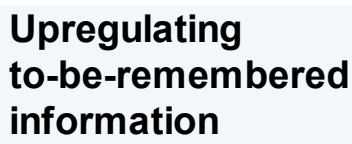

Selective encoding

Selective rehearsal

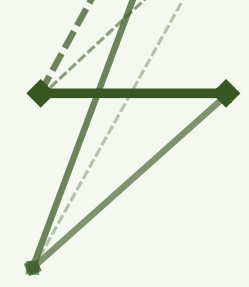

Combination

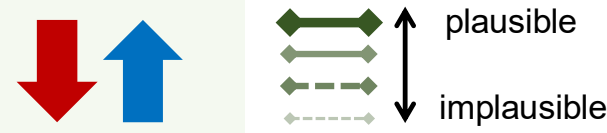

Note. Red curves represent the memory strength of to-be-forgotten items over serial position. Blue curves represent the memory strength of to-be-remembered items over serial position. Green lines connect processes that can, in combination, predict the serial position curves obtained in our experiments. The strength of the green lines reflects the plausibility of these combinations given our data. 
In the following, we will discuss five causal scenarios that could explain the present results, either through a single process in Figure 5, or a combination of two of these processes. We selected these five scenarios either because they are prominent explanations of item-wise directed forgetting in the episodic LTM, or because they are plausible given our data. We discuss for each scenario whether it can explain the overall difference in recognition performance between to-be-remembered and to-be-forgotten words, the invariance of that effect over manipulations of time (i.e., the SCI), as well as the serial position curves obtained in our additional analyses.

Selective Removal. In the first scenario, items are initially encoded into WM. Upon a forget cue, the last-presented item is selectively removed from WM, for instance via Hebbian anti-learning (Oberauer et al., 2012) or inhibition (e.g., Hasher \& Zacks, 1988; Zacks et al., 1996). Selective removal must be rapid as there is little time between a forget cue and the onset of the next word (see also Oberauer, 2018). On its own, such a removal process cannot explain the pattern of results in our serial position analyses: If removal operates fast and only on the lastpresented item, then binding memory for to-be-forgotten words should not change as a function of serial position.

Selective Encoding. In the selective encoding scenario, each new item is initially held in the focus of attention (Oberauer \& Kein, 2012). Only upon a remember cue, it is fully committed to WM by binding the item to its context. Upon a forget cue, the item is removed from the focus of attention without being fully encoded into WM. Such selective encoding of a to-beremembered item into WM has to proceed rapidly before the focus of attention is engaged by the next word. Like selective removal, on its own selective encoding does not account for the results of our serial position analyses. One could, however, assume that the encoding of each to-be- 
remembered item into WM depletes a limited WM resource (e.g., Popov \& Reder, 2020) resulting in a primacy gradient for to-be-remembered words as illustrated in Figure 5.

Selective Rehearsal and Decay. In the third scenario, all items are initially encoded into WM. Subsequently, selective rehearsal (e.g., MacLeod, 1975; Woodward et al., 1973) further strengthens items that are followed by a remember cue, whereas to-be-forgotten items are left to decay (e.g., Barrouillet et al., 2004; Ricker et al., 2016). Different from selective removal, decay affects all to-be-forgotten items non-selectively, and its effect increases as a function of time. Because items earlier in a list have more time to decay than items later in a list, decay naturally creates a recency gradient for all items. Furthermore, selective rehearsal accounts for the primacy effect in memory strength for to-be-remembered words, as early to-be-remembered words have more opportunity for rehearsal. At the same time, participants do not rehearse to-be-forgotten words. Thus, the beneficial effect of rehearsal for to-be-remembered words should mainly come from the primacy region of a list, which is in line with our findings. Together, decay and selective rehearsal produce serial positions curves similar to the ones we observed in our experiments.

Nevertheless, there are reasons to doubt this scenario. In addition to existing evidence against decay for verbal WM contents (Oberauer \& Lewandowsky, 2013), one aspect of our findings speaks against the decay and selective-rehearsal scenario: If to-be-forgotten items were simply left to decay over time, then a longer SCIs following every word would have given those items more time to decay. That should have led to stronger directed-forgetting effects with longer SCIs, which was not observed. Likewise, selective rehearsal would lead to a larger directedforgetting effect with longer SCIs, as this time could be used to strengthen earlier presented to- 
be-remembered items. Consequently, longer SCIs should result in a larger strength advantage of to-be-remembered over to-be-forgotten items, which we did not find.

Attentional Boost and Automatic Updating of WM. In the fourth scenario, all items are initially encoded into WM with some weak level of strength. Then, only upon a remember cue, individuals particularly attend to an item, thereby boosting its memory strength (e.g., Oberauer, 2003; Page \& Norris, 1998). It is suggested that the attention devoted to an item decreases with each successive item. If we additionally assume that participants have control over which item to boost by attending to it (as suggested by Oberauer \& Greve, in press), only to-be-remembered items receive an attentional boost. This creates a primacy effect for to-be-remembered but not tobe-forgotten words; nevertheless, to-be-forgotten words are still remembered to some extent, as they are encoded into WM with a baseline level of strength. This is exactly what we observed, and is in line with a similar observation made by Oberauer and Greve (in press).

The attentional-boost hypothesis on its own does not explain the recency effect on to-beforgotten items. This could be achieved by assuming that encoding of a new event into WM is accompanied by a general, automatic updating process that reduces the strength of all previous WM contents by a constant proportion. Such a default global updating process could serve to ensure that there is always enough WM capacity for encoding and maintaining new information. Such an automatic updating process creates a recency effect for to-be-forgotten items: At test, items presented later in a list were not subject to many updating steps, so that both to-beremembered as well as to-be-forgotten items presented in the recency part of a list are still remembered.

The idea of an attentional boost is similar to the selective-encoding assumption discussed above, when combined with the assumption of a partially depleted encoding resource. Hence, 
predictions made by the present scenario could also be obtained by assuming a selective encoding process that depletes a limited WM resource (e.g., Popov \& Reder, 2020), with one exception: With selective encoding of to-be-remembered items, the to-be-forgotten items are predicted to not be encoded into memory at all. Therefore, memory for to-be-forgotten items would be predicted to be at chance. The following scenario describes a possible way to avoid that limitation of the selective-encoding idea.

Selective Encoding, Incidental encoding into LTM, and Context Drift. In the fifth scenario, all items are incidentally encoded into LTM, with some weak level of strength. In line with many models of episodic LTM, this means that the items are associated to a temporal context (e.g., Brown et al., 2007; Davelaar et al., 2005; Sederberg et al., 2008). Only upon a remember cue, to-be-remembered items are additionally encoded into WM. Assuming that encoding into WM relies on a gradually depleted resource (Popov \& Reder, 2020) could explain the primacy effect for to-be-remembered items. Because the temporal context is constantly changing as new items (events) are encoded, the context at test is most similar to the most recent items. If the context at test is used as part of the retrieval cue, it cues the last-presented items the most, creating a recency gradient (see also Oberauer, 2003). If we assume that the encoding of associations between items and their temporal context occurs incidentally, unaffected by the intention to remember or forget, a recency gradient would be generated regardless of intention, hence should also be observed for to-be-forgotten words. As the directed-forgetting effect did not increase with longer SCIs, we further need to assume that the temporal context changes not as a function of the passage of time (as assumed, for instance, in the SIMPLE model of Brown et al., 2007), but as a function of events encoded into memory (as assumed, for instance, in the Temporal-Context Model of Sederberg et al., 2008). One limitation of the fifth scenario 
remains: It is unclear why the intention to remember or forget should not affect the encoding of item-context associations in episodic LTM (see Popov \& Dames for recent evidence that the intent to remember significantly boosts item-context associations in episodic LTM).

In conclusion, our results can only be explained by a combination of different processes: one process that explains why we observe a primacy gradient for to-be-remembered but not for to-be-forgotten words, and one process that explains why recent to-be-forgotten words are still remembered. Supported by recent work demonstrating that long-term directed-forgetting affects neural activity patterns of both to-be-remembered and to-be-forgotten items (Fellner et al., 2020), we therefore deem a combination of both up- and downregulatory processes likely. Of the suggested processes, two scenarios explain our data best: (1) the scenario involving automatic global updating of working memory in combination with an attentional boost or (2) the scenario involving incidental encoding of all items by binding them to a temporal context in episodic LTM, in combination with selective encoding of to-be-remembered items into WM. It is worth noting that in both suggested scenarios the memory cue triggers a rapid process to either selectively boost or encode the last-presented item into WM.

\section{Directed-forgetting in WM is Remarkably Robust}

We observed very effective forgetting in WM even when testing the to-be-forgotten items up to four times, and even when we announced upfront that to-be-forgotten items will occasionally be tested. Directed-forgetting effects in WM remained robust for cues delayed up to $5 \mathrm{~s}$. In the context of the attentional boost scenario, this implies that even after maintaining an item in WM for several seconds it can still be rapidly boosted. In the context of the selective encoding scenario, this means that an item can be held in the focus of attention for several seconds without committing it to WM, which then happens rapidly upon a remember cue. 


\section{Conclusion}

This work establishes a new method to investigate how the intention to remember or forget affects WM. Using this method, we demonstrate a strong impact of directed-forgetting on both item and binding memory in WM, as evident in participants' poor ability to access to-beforgotten words, and improved memory for to-be-remembered words. Directed-forgetting in WM is highly effective even after information has been held in memory for up to $5 \mathrm{~s}$. The present data can best be explained by a combination of two processes: One process renders memory representations of earlier events less accessible, and this happens as a function of subsequently encoded events, not as a function of time passing. Another process rapidly encodes or boosts memory strength only for those events that the person intends to remember.

\section{References}

Anderson, M. C., \& Hanslmayr, S. (2014). Neural mechanisms of motivated forgetting. Trends in Cognitive Sciences, 18(6), 279-292. https://doi.org/10.1016/j.tics.2014.03.002

Baayen, R. H., Davidson, D. J., \& Bates, D. M. (2008). Mixed-effects modeling with crossed random effects for subjects and items. Journal of Memory and Language, 59(4), 390412. https://doi.org/10.1016/j.jml.2007.12.005

Bancroft, T. D., Hockley, W. E., \& Farquhar, R. (2013). The longer we have to forget the more we remember: The ironic effect of postcue duration in item-based directed forgetting. Journal of Experimental Psychology: Learning, Memory, and Cognition, 39(3), 691-699. https://doi.org/10.1037/a0029523

Barrouillet, P., Bernardin, S., \& Camos, V. (2004). Time constraints and resource sharing in adults' working memory spans. Journal of Experimental Psychology: General, 133(1), 83-100. https://doi.org/10.1037/0096-3445.133.1.83 
Bjork, E. L., Bjork, R. A., \& Anderson, M. C. (1998). Varieties of goal-directed forgetting. Intentional Forgetting: Interdisciplinary Approaches., 103-137.

Bjork, R. A. (1970). Positive forgetting: The noninterference of items intentionally forgotten. Journal of Verbal Learning and Verbal Behavior, 9(3), 255-268. https://doi.org/10.1016/S0022-5371(70)80059-7

Brown, G. D. A., Neath, I., \& Chater, N. (2007). A temporal ratio model of memory. Psychological Review, 114(3), 539-576. https://doi.org/10.1037/0033295X.114.3.539

Bürkner, P.-C. (2018). Advanced bayesian multilevel modeling with the R package brms. The $R$ Journal, 10(1), 395. https://doi.org/10.32614/RJ-2018-017

Carpenter, B., Gelman, A., Hoffman, M. D., Lee, D., Goodrich, B., Betancourt, M., Brubaker, M., Guo, J., Li, P., \& Riddell, A. (2017). Stan: A probabilistic programming language. Journal of Statistical Software; Vol 1, Issue 1 (2017). https://doi.org/10.18637/jss.v076.i01

Dames \& Oberauer, 2021. (2021, August 24). Directed forgetting in working memory. osf.io/2vpfg

Davelaar, E. J., Goshen-Gottstein, Y., Ashkenazi, A., Haarmann, H. J., \& Usher, M. (2005). The Demise of Short-Term Memory Revisited: Empirical and Computational Investigations of Recency Effects. Psychological Review, 112(1), 3-42. https://doi.org/10.1037/0033295X.112.1.3

de Leeuw, J. R. (2015). jsPsych: A JavaScript library for creating behavioral experiments in a Web browser. Behavior Research Methods, 47(1), 1-12. https://doi.org/10.3758/s13428014-0458-y 
Ecker, U. K. H., Lewandowsky, S., \& Oberauer, K. (2014). Removal of information from working memory: A specific updating process. Journal of Memory and Language, 74, 77-90. https://doi.org/10.1016/j.jml.2013.09.003

Fawcett, J. M., \& Taylor, T. L. (2008). Forgetting is effortful: Evidence from reaction time probes in an item-method directed forgetting task. Memory \& Cognition, 36(6), 11681181. https://doi.org/10.3758/MC.36.6.1168

Fawcett, J. M., \& Taylor, T. L. (2012). The control of working memory resources in intentional forgetting: Evidence from incidental probe word recognition. Acta Psychologica, 139(1), 84-90. https://doi.org/10.1016/j.actpsy.2011.10.001

Fellner, M.-C., Waldhauser, G. T., \& Axmacher, N. (2020). Tracking selective rehearsal and active inhibition of memory traces in directed forgetting. Current Biology, 30(13), 26382644.e4. https://doi.org/10.1016/j.cub.2020.04.091

Festini, S. B., \& Reuter-Lorenz, P. A. (2013). The short- and long-term consequences of directed forgetting in a working memory task. Memory, 21(7), 763-777. https://doi.org/10.1080/09658211.2012.754900

Festini, S. B., \& Reuter-Lorenz, P. A. (2014). Cognitive control of familiarity: Directed forgetting reduces proactive interference in working memory. Cognitive, Affective, \& Behavioral Neuroscience, 14(1), 78-89. https://doi.org/10.3758/s13415-013-0231-1

Gronau, Q. F., Singmann, H., \& Wagenmakers, E.-J. (2018). bridgesampling: An R package for estimating normalizing constants. ArXiv:1710.08162 [Stat]. http://arxiv.org/abs/1710.08162 
Hasher, L., \& Zacks, R. T. (1988). Working memory, comprehension, and aging: A review and a new view. In Psychology of Learning and Motivation (Vol. 22, pp. 193-225). Elsevier. https://doi.org/10.1016/S0079-7421(08)60041-9

Hourihan, K. L., \& Taylor, T. L. (2006). Cease remembering: Control processes in directed forgetting. Journal of Experimental Psychology: Human Perception and Performance, 32(6), 1354-1365. https://doi.org/10.1037/0096-1523.32.6.1354

Judd, C. M., Westfall, J., \& Kenny, D. A. (2012). Treating stimuli as a random factor in social psychology: A new and comprehensive solution to a pervasive but largely ignored problem. Journal of Personality and Social Psychology, 103(1), 54-69. https://doi.org/10.1037/a0028347

Lee, Y.-S. (2013). Costs and Benefits in Item-Method Directed Forgetting: Differential Effects of Encoding and Retrieval. The Journal of General Psychology, 140(3), 159-173. https://doi.org/10.1080/00221309.2012.750591

Lewis-Peacock, J. A., Kessler, Y., \& Oberauer, K. (2018). The removal of information from working memory. Annals of the New York Academy of Sciences, 1424(1), 33-44. https://doi.org/10.1111/nyas.13714

MacLeod, C. M. (1975). Long-term recognition and recall following directed forgetting. Journal of Experimental Psychology: Human Learning and Memory, 1(3), 271-279. https://doi.org/10.1037/0278-7393.1.3.271

Macleod, C. M. (1999). The item and list methods of directed forgetting: Test differences and the role of demand characteristics. Psychonomic Bulletin \& Review, 6(1), 123-129. https://doi.org/10.3758/BF03210819 
Monsell, S. (1978). Recency, immediate recognition memory, and reaction time. Cognitive Psychology, 10(4), 465-501. https://doi.org/10.1016/0010-0285(78)90008-7

Muter, P. (1980). Very rapid forgetting. Memory \& Cognition, 8(2), 174-179. https://doi.org/10.3758/BF03213420

Oberauer, K. (2001). Removing irrelevant information from working memory: A cognitive aging study with the modified Sternberg task. Journal of Experimental Psychology: Learning, Memory, and Cognition, 27(4), 948-957. https://doi.org/10.1037/0278-7393.27.4.948

Oberauer, K. (2003). Understanding serial position curves in short-term recognition and recall. Journal of Memory and Language, 49(4), 469-483. https://doi.org/10.1016/S0749$596 \times(03) 00080-9$

Oberauer, K. (2009). Chapter 2 Design for a Working Memory. In Psychology of Learning and Motivation (Vol. 51, pp. 45-100). Elsevier. https://doi.org/10.1016/S00797421(09)51002-X

Oberauer, K. (2018). Removal of irrelevant information from working memory: Sometimes fast, sometimes slow, and sometimes not at all. Annals of the New York Academy of Sciences, 1424(1), 239-255. https://doi.org/10.1111/nyas.13603

Oberauer, K. (2019). Working memory capacity limits memory for bindings. Journal of Cognition, 2(1), 40. https://doi.org/10.5334/joc.86

Oberauer, K., \& Hein, L. (2012). Attention to information in working memory. Current Directions in Psychological Science, 21, 164-169. https://doi.org/10.1177/0963721412444727

Oberauer, K., \& Greve, W. (in press). Intentional Remembering and Intentional Forgetting in Working and Long-Term Memory. https://doi.org/10.1037/xge0001106 
Oberauer, K., \& Lewandowsky, S. (2013). Evidence against decay in verbal working memory. Journal of Experimental Psychology: General, 142(2), 380-411. https://doi.org/10.1037/a0029588

Oberauer, K., Lewandowsky, S., Awh, E., Brown, G. D. A., Conway, A., Cowan, N., Donkin, C., Farrell, S., Hitch, G. J., Hurlstone, M. J., Ma, W. J., Morey, C. C., Nee, D. E., Schweppe, J., Vergauwe, E., \& Ward, G. (2018). Benchmarks for models of short-term and working memory. Psychological Bulletin, 144(9), 885-958. https://doi.org/10.1037/bul0000153

Oberauer, K., Lewandowsky, S., Farrell, S., Jarrold, C., \& Greaves, M. (2012). Modeling working memory: An interference model of complex span. Psychonomic Bulletin \& Review, 19(5), 779-819. https://doi.org/10.3758/s13423-012-0272-4

Page, M. P. A., \& Norris, D. (1998). The primacy model: A new model of immediate serial recall. Psychological Review, 105(4), 761-781. https://doi.org/10.1037/0033295X.105.4.761-781

Popov, V., \& Dames, H. (2021, preprint). Intent Matters: Resolving the Intentional vs Incidental Learning Paradox in Episodic Long-term Memory. https://doi.org/10.31234/osf.io/jf2en

Popov, V., Marevic, I., Rummel, J., \& Reder, L. M. (2019). Forgetting is a feature, not a bug: Intentionally forgetting some things helps us remember others by freeing up working memory resources. Psychological Science, 30(9), 1303-1317. https://doi.org/10.1177/0956797619859531

Popov, V., \& Reder, L. M. (2020). Frequency effects on memory: A resource-limited theory. Psychological Review, 127(1), 1-46. https://doi.org/10.1037/rev0000161

Ricker, T. J., Nieuwenstein, M. R., Bayliss, D. M., \& Barrouillet, P. (2018). Working memory consolidation: Insights from studies on attention and working memory: An overview of 
working memory consolidation. Annals of the New York Academy of Sciences, 1424(1), 8-18. https://doi.org/10.1111/nyas.13633

Ricker, T. J., Vergauwe, E., \& Cowan, N. (2016). Decay Theory of Immediate Memory: From Brown (1958) to Today (2014). Quarterly Journal of Experimental Psychology, 69(10), 1969-1995. https://doi.org/10.1080/17470218.2014.914546

Sederberg, P. B., Howard, M. C., \& Kahana, M. J. (2008). A context-based theory of recency and contiguity in free recall. Psychological Review, 115, 893-912. https://doi.org/10.1037/a0013396

Williams, M., Hong, S. W., Kang, M.-S., Carlisle, N. B., \& Woodman, G. F. (2013). The benefit of forgetting. Psychonomic Bulletin \& Review, 20(2), 348-355. https://doi.org/10.3758/s13423-012-0354-3

Williams, M., \& Woodman, G. F. (2012). Directed forgetting and directed remembering in visual working memory. Journal of Experimental Psychology: Learning, Memory, and Cognition, 38(5), 1206-1220. https://doi.org/10.1037/a0027389

Woodward, A. E., Bjork, R. A., \& Jongeward, R. H. (1973). Recall and recognition as a function of primary rehearsal. Journal of Verbal Learning and Verbal Behavior, 12(6), 608-617. https://doi.org/10.1016/S0022-5371(73)80040-4

Zacks, R. T., Radvansky, G., \& Hasher, L. (1996). Studies of directed forgetting in older adults. Journal of Experimental Psychology: Learning, Memory, and Cognition, 22(1), 143-156. https://doi.org/10.1037/0278-7393.22.1.143 